\title{
Accuracy of different temperature reading techniques and associated stress response in hospitalized dogs
}

\author{
Samantha B. Gomart, DVM; Fergus J. W. Allerton, BSc, BVSc and Kris Gommeren, DVM, DECVIM
}

\begin{abstract}
Objective - To evaluate the accuracy and associated induced stress response of axillary, auricular, and rectal thermometry in hospitalized dogs.

Design - Prospective observational study from October 2011 to February 2012.

Setting - University veterinary teaching hospital.

Animals - Two hundred fifty hospitalized dogs. All hospitalized dogs were considered eligible unless their condition precluded measurement at one of the designated sites.

Interventions - A veterinary auricular infrared device for auricular temperature (OT) and an electronic predictive thermometer for rectal temperature (RT) and axillary temperature (AT) were used for temperature measurements. All recordings were obtained by the same investigator in a randomized fashion. Heart rate was noted before and immediately after each measurement. Stress behaviors (eg, vocalization, lip licking, shaking, panting, defensive behavior) were also recorded and graded from 0 (lowest) to 4 (highest). Signalment, analgesic therapy, and length of hospitalization were recorded.

Measurements and Main Results - RT measurements were associated with greatest increase in heart rate $(P<0.05)$. Scores obtained for defensive behavior, lip licking, and vocalization were lowest with AT and highest with RT measurements $(P<0.05)$.

Mean RT, AT, and OT were $38.0^{\circ} \mathrm{C}\left(\mathrm{SD}: 0.85^{\circ} \mathrm{C}\right), 37.0^{\circ} \mathrm{C}\left(\mathrm{SD}: 0.99^{\circ} \mathrm{C}\right)$, and $37.23^{\circ} \mathrm{C}\left(\mathrm{SD}: 1.0382^{\circ} \mathrm{C}\right)$, respectively. AT and OT were moderately correlated with RT ( $r=0.70$ and $r=0.64$, respectively). Gender $(P=0.02)$ and coat length $(P=0.03)$ had a significant influence on results. No effect of dehydration, body condition, analgesia, age, reproductive status, or operator experience was observed $(P>0.05)$.

Conclusions - AT and to a lesser extent OT are reliable, less stressful alternatives to estimate RT in dogs. Further studies are needed to evaluate these techniques in hyperthermic dogs, and to evaluate the use of AT and OT as monitoring tools in intensive care patients.
\end{abstract}

(J Vet Emerg Crit Care 2014; 24(3): 279-285) doi: 10.1111/vec.12155

Keywords: axilla, behavior, body temperature, ear, rectum, thermometers

\section{Introduction}

Temperature measurement (TM) is an essential part of any clinical examination in veterinary medicine. Significant deviations from the normal temperature range may serve as an early indicator of a change in the patient's condition that may be related to the presence

From the Department of Clinical Sciences, Small Animal Medicine, Faculty of Veterinary Medicine, University of Liège, Liège, Belgium.

The authors declare no conflict of interests.

Offprints will not be available from the authors.

Presented as a poster at the 11th Congress of the European Veterinary Emergency and Critical Care Society in Barcelona in June 2012.

Address correspondence and reprint requests to Dr. Samantha Gomart, CVU, 20 Blvd de Colonster, 4000 Liège, Belgium. Email: Samantha. gomart@gmail.com

Submitted June 05, 2012; Accepted December 21, 2013.

\section{Abbreviations}

AT axillary temperature

HRE heart rate elevation

OT auricular temperature

RT rectal temperature

TM temperature measurement

of infection, systemic inflammatory response syndrome, immune-mediated diseases, neoplasia, or shock..$^{1-3}$

Direct measurement of the core body temperature is the recognized gold standard technique. However, direct measurement requires an invasive approach and therefore in not clinically applicable. In the majority of cases, predictive rectal thermometry is reliable and 
provides an accurate estimation of core body temperature in dogs. ${ }^{4,5}$ Digital predictive thermometers measure the rate of temperature change during the first few seconds and mathematically predict the final temperature within 15 seconds.

However, obtaining a rectal temperature (RT) can be stressful for the patient. Insertion of a rectal thermometer can elicit defensive behavior from the patient, especially if the animal is hospitalized for a prolonged length of time and requires regular temperature monitoring, such as is the case for many intensive care patients. Rectal thermometers have been implicated in the spread of infection in human hospital wards. ${ }^{6}$ It may also be contraindicated in patients with wounds or surgical conditions affecting the anal region. In individuals with these conditions, an alternative means of TM would be required.

In human medicine, the axillary site is a frequently used alternative to rectal thermometry as the risk of cross-contamination is decreased; it is convenient and less embarrassing for the patient. Studies in human medicine have shown that axillary temperature (AT) was influenced by body mass, tissue insulation, vasoconstriction, gender, age, and by the thermal environment. ${ }^{7-9} \mathrm{To}$ the authors' knowledge, comparison of AT and RT readings has not previously been reported in dogs.

Infrared auricular thermometry is gaining popularity in veterinary medicine because it offers improved patient compliance, and quicker results than is provided by a rectal thermometer. ${ }^{10-12}$ Auricular thermometers use infrared technology to measure the heat emanating from the tympanic membrane. The tympanic membrane shares vascularization with the hypothalamus via the carotid artery; auricular temperature (OT) readings are thus expected to closely reflect core temperature. ${ }^{4}$ A study on dogs with otitis externa of varying severity showed that this type of measurement was not influenced by local inflammation ${ }^{12}$ in contradiction with the findings of a previous study showing that otitis externa influenced ear canal temperature. ${ }^{13}$ It has been reported that human aural thermometers demonstrate considerable inaccuracy in animals, most likely due to difficulties in accessing the tympanic membrane attributable to the differences in ear conformation between humans and dogs. ${ }^{10}$ Previous studies have reported conflicting results regarding the accuracy of auricular thermometers designed for veterinary use. $4,5,10,12,14,15$

The purpose of our study was to evaluate the induced stress response and the accuracy of axillary, auricular, and rectal thermometry in a large number of hospitalized dogs.

We hypothesized that measuring AT and OT would be would be less stressful for the patient compared to RT measurement, while maintaining reliability of the results. Patient characteristics including signalment, hy- dration status, and the nature of any ongoing analgesic medication were also recorded as these factors have potential to influence the dog's temperature. ${ }^{16,17}$

\section{Materials and Methods}

A prospective single center, single operator study was conducted. A single investigator performed all data collection. All dogs hospitalized between October 2011 and February 2012 were considered eligible; each dog was only evaluated once during its hospitalization.

Gender, sterilization status, age, body condition, hair coat length, hydration status, length of hospitalization, and recent analgesic administration were recorded. Gender and sterilization status (entire or neutered) were noted. The age (in years) was reported and for comparative purposes, dogs were grouped as puppies $(<1)$, young adults (1-6), and seniors $(>6)$. Body condition (BC) was scored from 1 (emaciated) to 9 (severely overweight). For statistical analysis, we divided the scores into categories $(\mathrm{BC}<4$ : lean, $4 \leq \mathrm{BC}<6$ : normal, $\mathrm{BC} \geq 6$ : overweight). Hair coat was classified as long, short, or curly. Hydration status was assessed based on the moistness of oral mucous membranes, skin turgor, and degree of enophthalmia. All animals clinically suspected to be at least $5 \%$ dehydrated were considered dehydrated. The length of hospitalization was described as short ( 1 day), moderate ( 2 days), and long ( $>2$ days). We noted all analgesic treatment administered to the dog during hospitalization and active at the time of measurement. For comparative purposes, we divided the analgesic treatments into categories: none, nonsteroidal antiinflammatory drugs, opioids, other (other categories of analgesics or combination of analgesics).

Dogs were allowed acclimatization periods of at least 30 minutes following admittance onto the hospitalization ward before TM were performed.

Dogs were excluded if their condition precluded TM at 1 of the designated sites, due to severe bilateral ear disease, perianal wounds, or bilateral axillary skin disease.

The order of RT, AT, and OT measurement for each dog was preset in a random manner. The heart rate was recorded before and immediately after each TM. Heart rate elevation (HRE) was defined as the percentage change in heart rate between the 2 measurements. A cool down period of 30 seconds was observed in between TM at each site.

In order to assess the stress induced by each TM, manifestations of stress behavior were recorded. Vocalization, lip licking, shaking, panting, and defensive behavior were noted according to the following predefined criteria. Each behavior was graded from 0 (absent) to 
Table 1: Scoring system used for stress behavior during temperature measurements

\begin{tabular}{|c|c|c|c|c|c|}
\hline Score & Panting & Shaking & Licks its lips & Vocalization & Defensive behavior \\
\hline 0 & None & None & 0 & No vocalization & Dog unconcerned by TM \\
\hline 1 & $<2 \sec$ & $<2 \mathrm{sec}$ & 1 time & Whines once & $\begin{array}{l}\text { Dog looks towards observer but } \\
\text { remains still }\end{array}$ \\
\hline 2 & $\begin{array}{c}>2 \text { sec }, \leq 25 \% \\
\text { total time }\end{array}$ & $\begin{array}{c}>2 \text { sec }, \leq 25 \% \\
\text { total time }\end{array}$ & 2 times & Whining $\leq 25 \%$ total time & $\begin{array}{l}\text { Tries to get away from the observer } \\
\text { but restraint possible without } \\
\text { difficulty }\end{array}$ \\
\hline 3 & $\begin{array}{c}>25 \%, \leq 50 \% \\
\text { total time }\end{array}$ & $\begin{array}{c}>25 \%, \leq 50 \% \\
\text { total time }\end{array}$ & 3 times & $\begin{array}{l}\text { Growling/whining }>25 \% \\
\text { of total time }\end{array}$ & $\begin{array}{l}\text { Restraint difficult and dog moves to } \\
\text { limit access to TM site }\end{array}$ \\
\hline 4 & $\geq 50 \%$ total time & $\geq 50 \%$ total time & $\geq 4$ times & Barking, screaming & $\begin{array}{l}\text { Dog attempts to bite, restraint } \\
\text { impossible by a single observer }\end{array}$ \\
\hline
\end{tabular}

4 (most significant manifestation of relevant behavior) (Table 1).

An auricular infrared veterinary device ${ }^{a}$ was used for OT. A disposable plastic sheath ${ }^{\mathrm{b}}$ made for the device was fitted onto the probe prior to TM, and in accordance with the manufacturer's instructions, the ear was manipulated to straighten the ear canal and allow a direct alignment of the probe with the tympanic membrane. An electronic predictive thermometer ${ }^{\mathrm{c}}$ was used for rectal and axillary measurements. For rectal measurement, the thermometer was prelubricated with lubricating jelly. The thermometer was inserted approximately $3 \mathrm{~cm}$ into the rectum and held against the rectal mucosa until an endpoint reading audible beep was heard. For AT, the thermometer was placed approximately $3 \mathrm{~cm}$ in the axilla, the tip of it placed against the skin with the limb held close to the body, until an endpoint reading audible beep was heard. After each measurement, the thermometer was cleaned with a gauze swab and disinfected with an antiseptic solution for cutaneous use (ethanol $96 \%$-ether). OT and AT were measured in only one ear, which was dependent on the positioning of the animal to minimize patient disturbance, unless that side displayed severe signs of disease.

\section{Statistical Analysis}

A mixed linear model ${ }^{\mathrm{d}}$ was applied to compare HRE for the different measurement sites. The measurement site and the rank of TM were included as a fixed effect. The dog was included as a random effect. Chi-square tests were used to evaluate the effect of different TM on the stress behavior and the effect of the length of hospitalization (therefore multiple rectal measurements) on stress behavior for rectal thermometry.

Column graphics were used to provide visual assessment of stress behavior scores for each of the TM. Readings from the auricular and axillary devices were compared with RT. A mixed model approach was used. The type of TM, the coat length, gender, rank of the TM, hy- dration status, analgesia, age category, body condition, and analgesia were included as fixed effects. The dog was included as a random effect. For all measurements, a value of $P<0.05$ was considered significant.

Though the correlation coefficient $(r)$ is often used in similar studies, it is not a statistically appropriate analysis when comparing 2 methods of measurement. ${ }^{18}$ Nevertheless, for clinical purposes, calculation between the types of TM was made using a Pearson's productmoment correlation coefficient. Scatter plots were used to provide visual assessment of the correlation between the TM.

\section{Results}

This prospective study included 250 dogs, of 71 different breeds (34 Mix breed dogs, 21 Labrador Retrievers, 10 Golden Retrievers, 8 Bernese Mountain dogs, 8 Chihuahuas, 8 Jack Russels, 8 Yorkshire Terriers, 7 Beagles, 7 Border Collies, 7 Dachshunds, 7 German Shepherds, 7 Rottweilers, 6 American Staffordshire Terriers, 6 Cavalier King Charles Spaniels, 6 English Cocker Spaniels, 5 Belgian Malinois, 5 English Bulldogs, 5 Shih Tzus, and 54 other breeds). These dogs were hospitalized for various purposes in the teaching hospital ranging from postoperative elective surgery to long-term hospitalized patients with life-threatening illness.

HRE was higher with RT than other measurement techniques. Taking the rectal measurement as a baseline, HRE was $15.2 \%$ less for the axillary measurement (SD = 2.6, $P<0.001)$ and $6 \%$ less for the auricular measurement $(\mathrm{SD}=2.6, P=0.013)$. The rank of TM did not have an effect on HRE.

Defensive behavior, lip licking, and vocalization were significantly different for each TM $(P<0.05)$. Figure 1 illustrates stress behavior scores obtained for each TM. Scores obtained for defensive behavior, vocalization, and lip licking, were lowest with axillary measurements. Highest scores were associated with rectal measurements. 

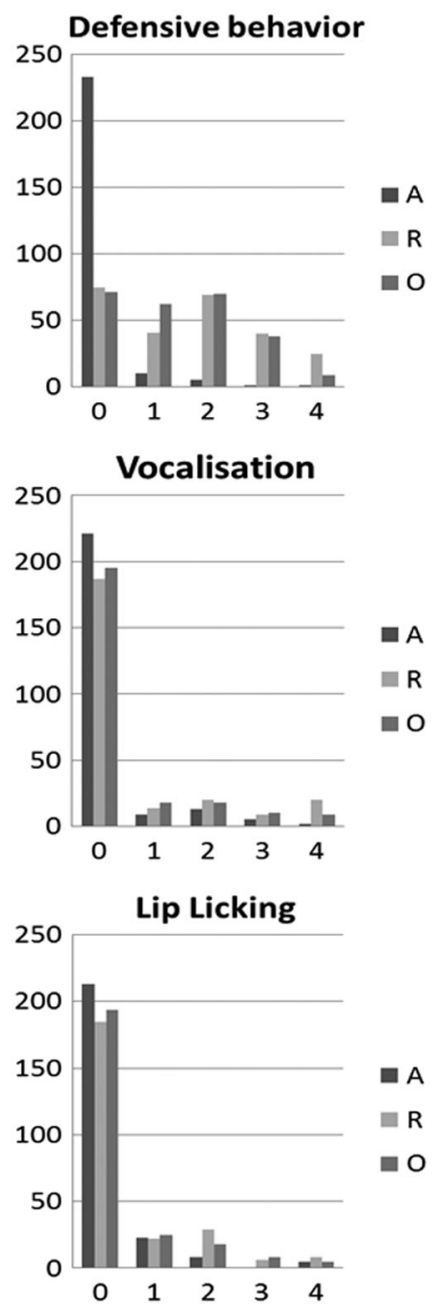

Figure 1: Distribution of stress scores for A (axillary), R (rectal), and $\mathrm{O}$ (auricular) temperature measurement.

Duration of hospitalization and incidence of panting and shaking were not significantly different. The operator's experience and the rank of TM did not have an effect on TM.

Overall, mean RT was $38.0^{\circ} \mathrm{C}$ (SD: $0.85^{\circ} \mathrm{C}$ ), and ranged from 35 to $40.4^{\circ} \mathrm{C}$. Mean AT was $37.0^{\circ} \mathrm{C}$ (SD: $1.0^{\circ} \mathrm{C}$ ), and ranged from 33.4 to $39.3^{\circ} \mathrm{C}$. Mean OT was $37.2^{\circ} \mathrm{C}$ (SD: $1.0^{\circ} \mathrm{C}$ ) ranging from 34.3 to $39.6^{\circ} \mathrm{C}$. Correlations between different TM techniques are presented in Figure 2. The mixed model approach showed that the RT could be approximated from AT or OT. The mean temperature difference between RT and AT was $1.0^{\circ} \mathrm{C}$ (SD: $0.1^{\circ} \mathrm{C}, P<$ $0.001)$. The mean RT could be approximated by adding $0.6^{\circ} \mathrm{C}$ (SD: $\left.0.06^{\circ} \mathrm{C}, P<0.001\right)$ to OT $(P<0.0001)$. Certain physical characteristics reduce the differences between RT and other types of TM. Female gender was associated with a smaller difference between RT and both OT and AT $(P=0.024)$. Similarly long hair coat was associ- ated with a smaller difference between RT and other TM approaches $(P=0.030)$. Correction factors that need to be applied to derive RT from AT or OT were calculated (Table 2). No effect of body condition, age, or reproductive status was observed. Hydration status and analgesia did not influence TM.

\section{Discussion}

Rectal thermometry is the standard method of temperature measurement in clinical practice. However, it can be associated with significant stress for the patient. In aggressive patients, it may incur significant risks of injury to personnel. The availability of an alternative approach may be in both the animal's and the operator's interest. Having a less stressful alternative to rectal thermometry can enable closer monitoring of patients. This study showed that there are alternatives methods of temperature measurement that are less stressful for the hospitalized dogs.

Heart rate variation is considered a good indicator for the noninvasive assessment of autonomic nervous system activity in response to psychophysiological stress. ${ }^{19}$ In our study, the finding that HRE is greatest following rectal measurement supports that this method is the most stressful way of TM while axillary thermometry was the least stressful. Many other factors can modulate heart rate in a short period of time including changes in posture and locomotion. ${ }^{20}$ For this reason, we also recorded behavioral data to extend the assessment of stress. In this study, evidence of increased scores obtained for defensive behavior, lip licking, and vocalization correlated with HRE findings. This supported our contention, that the greatest stress was induced by rectal thermometry while axillary thermometry was the least stressful for the animal. Previous studies on stressed dogs reported increased vocalization, panting, and trembling. ${ }^{21}$ Increased body shaking and lip licking have also been reported to constitute behavioral indications of acute stress in $\operatorname{dog} .{ }^{22}$ A limitation of this study is that even if behavioral parameters may help to identify acute stress in the dog, they remain a subjective assessment and may be misinterpreted.

Our study showed that AT was moderately correlated with RT $(r=0.70)$. A weaker correlation of 0.64 was found between OT and RT, in agreement with a previously reported feline study. ${ }^{14}$ However, several earlier studies found much higher degrees of correlation between RT and OT.5,10,12 These latter studies described temperature measurement in specific conditions and involved smaller and more homogenous subsets of dogs, and thus may be less applicable to wider populations of dogs. Similarly conflicting results regarding accuracy of auricular thermometry 


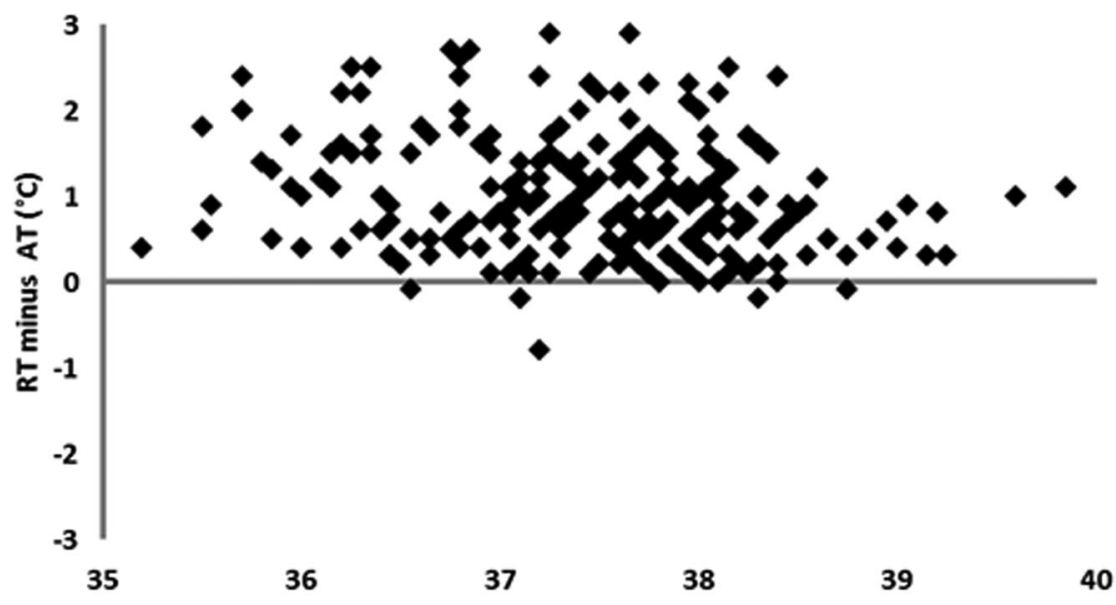

Average of RT and AT $\left({ }^{\circ} \mathrm{C}\right)$
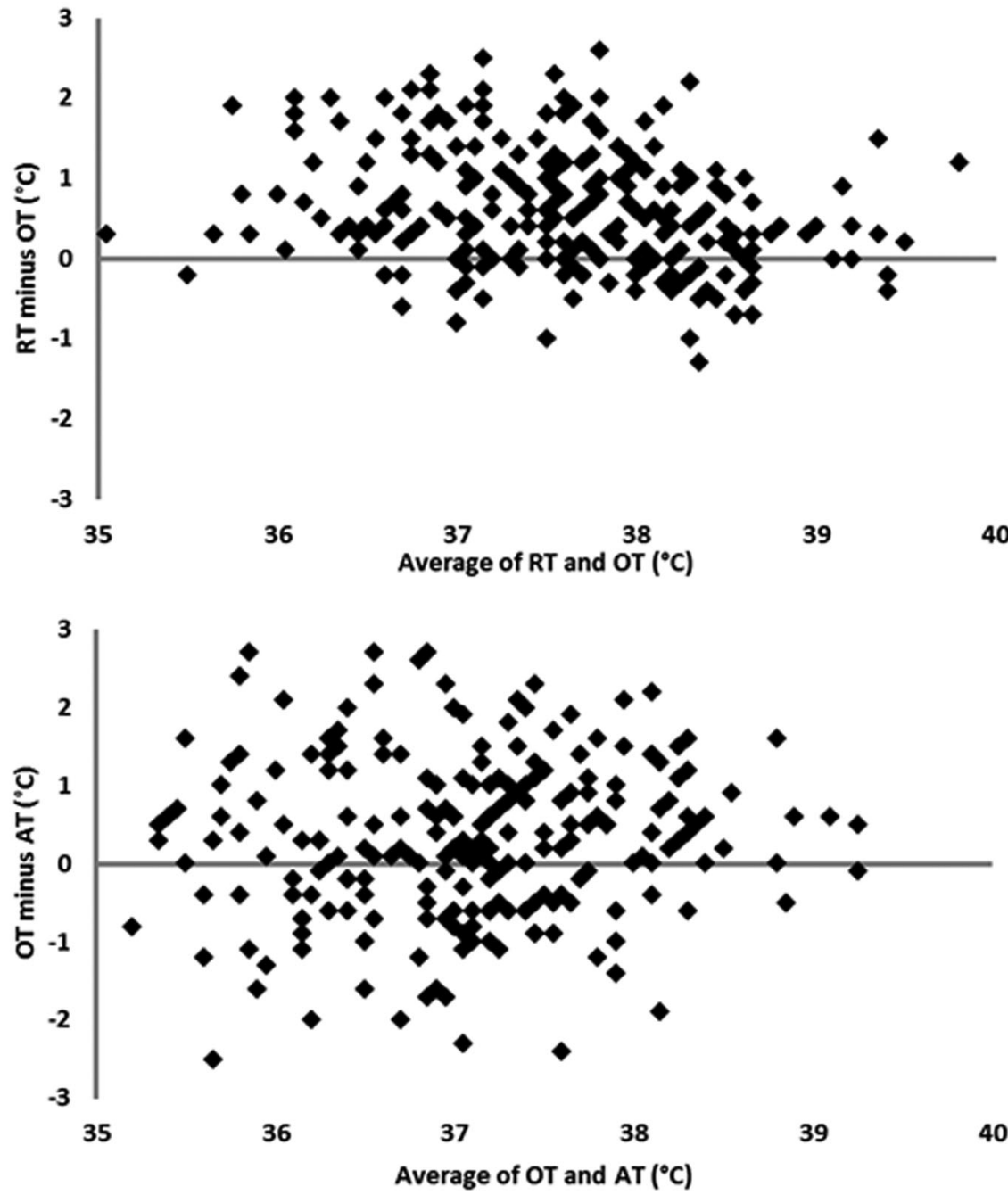

Figure 2: For each plot, the Y-axis represents the difference between two sites of temperature measurements while the X-axis represents the average of the temperature measured at the two sites. The reference line shown corresponds to zero differences (ie, if the reading from the described location matched the other reading site perfectly, then all points would fall on this line). RT - rectal temperature; $\mathrm{AT}$ - axillary temperature; OT - auricular temperature. Temperatures are in ${ }^{\circ} \mathrm{C}$. 
Table 2: Correction factors to derive rectal temperature from axillary temperature or auricular temperature. AT = axillary temperature; OT $=$ auricular temperature

\begin{tabular}{lll}
\hline Signalment & AT & OT \\
\hline Female with curly or short hair coat & $+0.8^{\circ} \mathrm{C}$ & $+0.4^{\circ} \mathrm{C}$ \\
Female with long hair coat & $+0.6^{\circ} \mathrm{C}$ & $+0.2^{\circ} \mathrm{C}$ \\
Male with curly or short hair coat & $+1^{\circ} \mathrm{C}$ & $+0.6^{\circ} \mathrm{C}$ \\
Male with long hair coat & $+0.8^{\circ} \mathrm{C}$ & $+0.4^{\circ} \mathrm{C}$ \\
\hline
\end{tabular}

were obtained in studies of humans, cats, and other species. ${ }^{14,15,23-25}$

Previous studies have shown auricular thermometry to be more accurate at hypothermic than hyperthermic temperatures in dogs and cats and humans. ${ }^{4,5,9,14}$ In our study, the correlation between OT and RT did not improve for dogs in a hypothermic state. The majority of dogs in this study were either hypothermic or normothermic. Further studies are needed with febrile patients to determine the accuracy of AT and OT at higher temperatures.

The dogs in this study presented a range of conditions that provoked normal, elevated, or decreased core body temperatures. Nevertheless the mean temperatures in our study correspond to the temperatures described in earlier studies even if the wider range in our temperatures reflects the heterogeneous population examined. The mean RT $\left(38.0^{\circ} \mathrm{C}\right)$ reported here is similar to previously reported normal canine RT $\left(38.0-39.0^{\circ} \mathrm{C}\right) .^{26}$

Our study found a mean OT of $37.2^{\circ} \mathrm{C}$. A previous study on 40 healthy dogs using infrared veterinary auricular thermometry reported a mean OT of $37.9^{\circ} \mathrm{C}$. $^{10} \mathrm{To}$ our knowledge there is no reported range for AT in dogs.

Although a veterinary device was used in our study to limit underestimation of OT due to anatomic difference in the ear canals of dogs and humans, ${ }^{10,11,27}$ mean OT was consistently lower than RT in our study. This finding matched those of previous studies using veterinary auricular devices. ${ }^{4,10}$ Some of the differences observed may reflect operator error since correct positioning of the probe is of paramount importance to obtain an accurate measure of the temperature of the tympanic membrane. Several studies documented a very weak correlation between duplicate measurements performed by different observers. $^{4,27}$ In our study, all measurements were realized by a single investigator to minimize the distortion induced by changing operator. Operator experience was shown to not have any effect on the accuracy of auricular measurement. This contradicts a prior study in human medicine that demonstrated an effect of training on the quality of the measurement. ${ }^{9}$ When performed by a single observer the auricular method has been shown to be repeatable. ${ }^{5}$ In the present study, no readings were repeated on any individual patient and only a single au- ricular measure was taken. Further studies are needed to evaluate if AT and OT curves follow RT curves and could be a reliable monitoring tool.

AT also underestimated RT in our study. The relationship between AT and RT has been investigated in human medicine. In a large systematic review comparing AT and RT in children and young people, ${ }^{28}$ the mean temperature difference (RT minus AT) for electronic thermometers was $0.85^{\circ} \mathrm{C}$ in children. In 2004, an equation was derived to calculate RT from $\mathrm{AT}^{29}$ : Rectal temperature $\left({ }^{\circ} \mathrm{C}\right)=0.98 \times$ axillar temperature $\left({ }^{\circ} \mathrm{C}\right)+0.8^{\circ} \mathrm{C}$.

In our study, we also established an equation to estimate RT from OT and AT, and furthermore we identified correction factors for gender and coat length. Indeed, in our study, females had a higher mean RT, OT, and AT. A previous study realized on 650 dogs reported that sex did not have an influence on OT. ${ }^{11}$

Several studies in human medicine showed significantly higher RT, OT, and AT in women. ${ }^{9,25}$ The dissimilarity between genders has been explained by the fact that women have a thicker layer of subcutaneous fat, insulating the body from heat loss. ${ }^{30}$ Also, the menstrual cycle has been reported to alter body temperature in premenopausal women. ${ }^{9}$ Even though we did not consider the influence of ovulation, we found that neuter status did not have a significant effect.

Furthermore, we found that OT, AT, and RT were higher in long-haired dogs. Interestingly, it has been shown that dogs with hirsute ear canals had significantly lower OT than dogs with hairless ear canals. ${ }^{11}$ This variable was not investigated in our study.

The different readings obtained by auricular or axillary thermometry may be erroneous and relate to systematic limitations in these measurement devices, but might also reflect actual temperature differences at different sites within the body. ${ }^{31}$ It is important to realize that body temperature varies by location. The rectal area has a low blood flow and high insulation and, therefore, the temperature is higher than at other places during a steady state. ${ }^{32}$ The ear temperature, sharing the same vascular supply that perfuses the hypothalamus, rapidly reacts to temperature changes. ${ }^{33}$ As the rectum and armpits have no thermal significance of their own, the temperature at these sites changes more slowly and, therefore, lags behind the ear temperature, especially during rapid thermal change. ${ }^{34}$

\section{Conclusions}

Our study showed axillary and auricular thermometry are reliable, less stressful alternatives to estimate RT in dogs. AT and OT are however influenced by factors such as coat length and gender. Further studies are needed to evaluate these techniques in hyperthermic dogs in 
particular, and to evaluate if AT and OT curves follow RT curves and could be considered reliable monitoring tools in intensive care patients.

\section{Acknowledgments}

The authors would like to thank Dr. Marc Vandenheede for his advice regarding stress behavior evaluation, and Dr. Jean Marie Bedouin for his help in statistical analysis.

\section{Footnotes}

a Pet-Temp PT-300, Advanced Monitors Corporation, San Diego, CA.

b Pet-Temp LC-40 Advanced Monitors Corporation.

c Thermoval rapid flex - PAUL HARTMANN, Dubai, United Arab Emirates.

d R Development Core Team (2011), R Foundation for Statistical Computing, Vienna, Austria.

\section{References}

1. Nelson RW, Couto CG. Fever of undertermined origin, In: Nelson RW, Couto CG. eds. Small Animal Internal Medicine, 2nd edn. St. Louis: Mosby Inc.; 1998, pp. 1231-1235.

2. Hauptman JG, Walshaw R, Olivier NB. Evaluation of the sensitivity and specificity of diagnostic criteria for sepsis in dogs. Vet Surg 1997; 26(5):393-397.

3. Brady CA, Otto CM. Systemic inflammatory response syndrome, sepsis, and multiple organ dysfunction. Vet Clin North Am Small Anim Pract 2001; 31(6):1147-1162.

4. Greer RJ, Cohn LA, Dodam JR, et al. Comparison of three methods of temperature measurement in hypothermic, euthermic, and hyperthermic dogs. J Am Vet Med Assoc 2007; 230(12):1841-1848.

5. Southward E, Mann F, Dodam JR, et al. A comparison of auricular, rectal and pulmonary artery thermometry in dogs with anesthesia induced hypothermia. J Vet Emerg Crit Care 2006; 16(3):172175.

6. French GL. Salmonella cross-infection associated with contamination of rectal thermometers. J Hosp Infect 1981; 2(4):389-390.

7. Bhandari V, Narang A. Thermoregulatory alterations as a marker for sepsis in normothermic premature neonates. Indian Pediatr 1992; 29(5):571-575.

8. Collins KJ, Dore C, Exton-Smith AN, et al. Accidental hypothermia and impaired temperature homoeostasis in the elderly. Br Med J 1977; 1(6057):353-356

9. Sund-Levander M, Grodzinsky E, Loyd D, et al. Errors in body temperature assessment related to individual variation, measuring technique and equipment. Int J Nurs Pract 2004; 10(5):216-223.

10. Rexroat J, Benish, K, Fraden J. Clinical Accuracy of Vet-Temp Instant Ear Thermometer: Comparative Study with Dogs and Cats. San Diego: Advanced Monitors Corporation; 1999, http:/ / www.admon. com/wp-content/uploads/2010/09/Humane-Society-WhitePaper.pdf.

11. Huang HP, Huang HM. Effects of ear type, sex, age, body weight, and climate on temperatures in the external acoustic meatus of dogs. Am J Vet Res 1999; 60(9):1173-1176.

12. Gonzalez AM, Mann FA, Preziosi DE, et al. Measurement of body temperature by use of auricular thermometers versus rectal thermometers in dogs with otitis externa. J Am Anim Hosp Assoc 2002; 221(3):378-380.

13. Huang HP, Shih HM. Use of infrared thermometry and effect of otitis externa on external ear canal temperature in dogs. J Am Vet Med Assoc 1998; 213(1):76-79.
14. Kunkle GA, Nicklin CF, Sullivan-Tamboe DL. Comparison of body temperature in cats using a veterinary infrared thermometer and a digital rectal thermometer. J Am Anim Hosp Assoc 2004; 40(1):4246.

15. Goodwin S. Comparison of body temperatures of goats, horses, and sheep measured with a tympanic infrared thermometer, an implantable microchip transponder, and a rectal thermometer. Contemp Top Lab Anim Sci 1998; 37(3):51-55.

16. Kuo WC, Keegan RD. Comparative cardiovascular, analgesic, and sedative effects of medetomidine, medetomidine-hydromorphone, and medetomidine-butorphanol in dogs. Am J Vet Res 2004; 65(7):931-937.

17. Pypendop B, Verstegen J. A comparison of the sedative and analgesic effects of buprenorphine in combination with acepromazine, midazolam or medetomidine in dogs. Vet Anaesth Analg 1994; 21(1):1520.

18. Bland JM, Altman DG. Statistical methods for assessing agreement between two methods of clinical measurement. Lancet 1986; 1(8476):307-310.

19. Tiller WA, McCraty R, Atkinson M. Cardiac coherence: a new, noninvasive measure of autonomic nervous system order. Altern Ther Health Med 1996; 2(1):52-65.

20. Vincent IC, Michell AR. Relationship between blood pressure and stress-prone temperament in dogs. Physiol Behav 1996; 60(1):135138.

21. Solomon RL, Kamin LJ, Wynne LC. Traumatic avoidance learning: the outcomes of several extinction procedures with dogs. J Abnorm Psychol 1953; 48(2):291-302.

22. Beerda B, Schilder M, Van Hooff J, et al. Behavioural, saliva cortisol and heart rate responses to different types of stimuli in dogs. Appl Anim Behav Sci 1997; 58(1998):365-381.

23. Michaud A. Comparison of an infrared thermometer to rectal thermometers in cats. Feline Pract 1996; 24(6):25-30.

24. Dodd SR, Lancaster GA, Craig JV, et al. In a systematic review, infrared ear thermometry for fever diagnosis in children finds poor sensitivity. J Clin Epidemiol 2006; 59(4):354-357.

25. Chamberlain JM, Terndrup TE, Alexander DT, et al. Determination of normal ear temperature with an infrared emission detection thermometer. Ann Emerg Med 1995; 25(1):15-20.

26. Rijnberk A. General examination, In: Rijnberk A, Van Sluijsfj FJ. eds. Medical History and Physical Examination in Companion Animals Philadelphia: Elsevier Ltd.; 2009, p. 54.

27. Sousa MG, Carareto R, Pereira-Junior VA, et al. Comparison between auricular and standard rectal thermometers for the measurement of body temperature in dogs. Can Vet J 2011; 52(4):403-406.

28. Craig JV, Lancaster GA, Williamson PR, et al. Temperature measured at the axilla compared with rectum in children and young people: systematic review. BMJ 2000; 320(7243):1174-1178.

29. Chaturvedi D, Vilhekar KY, Chaturvedi P, et al. Comparison of axillary temperature with rectal or oral temperature and determination of optimum placement time in children. Indian Pediatr 2004; 41(6):600-603.

30. Fox RH, Lofstedt BE, Woodward PM, et al. Comparison of thermoregulatory function in men and women. J Appl Physiol 1969; 26(4):444-453.

31. Cunningham JG. Thermoregulation, In: Cunningham JG. ed. Textbook of Veterinary Physiology, 3rd edn. Philadelphia: WB Saunders Co; 2002, p. 537.

32. Eichna LW, Berger AR, Rader B, et al. Comparison of intracardiac and intravascular temperatures with rectal temperatures in man. Clin Investig 1951; 30(4):353-359.

33. Boulant J. Neural thermal reception and regulation of body temperature, In: Blatteis CM. ed. Physiology and Pathophysiology of Temperature Regulation. Singapore: World Scientific Publishing; 1998, pp. 94-105.

34. Blatteis CM. Temperature regulation in special situations, In: Blatteis CM. ed. Physiology and Pathophysiology of Temperature Regulation. Singapore: World Scientific Publishing; 1998, pp. 260-271. 